\title{
Average biometry of the cornea in a large population of Iranian school children
}

\author{
Rafael Navarro ${ }^{1 *}$, Jos Rozema ${ }^{2,3}$, Mohammad Hassan Emamian ${ }^{4}$, \\ HASSAN HASHEMI ${ }^{5}$ AND AKBAR FOTOUHI ${ }^{6}$ \\ ${ }^{I} I C M A$, Consejo Superior de Investigaciones Cientificas \& Universidad de Zaragoza, Facultad de \\ Ciencias. Zaragoza, Spain \\ ${ }^{2}$ Volantis, Department of Ophthalmology, Antwerp University Hospital, Edegem, Belgium \\ ${ }^{3}$ Department of Medicine and Health Sciences, Antwerp University, Wilrijk, Belgium \\ ${ }^{4}$ Ophthalmic Epidemiology Research Center, Shahroud University of Medical Sciences, Shahroud, Iran \\ ${ }^{5}$ Noor Research Center for Ophthalmic Epidemiology, Noor Eye Hospital, Tehran, Iran \\ ${ }^{6}$ Department of Epidemiology and Biostatistics, School of Public Health, Tehran University of Medical \\ Sciences, Tehran, Iran \\ *rafaelnb@unizar.es
}

\begin{abstract}
This work establishes the average Scheimpflug corneal tomography for a population of 4953 healthy Iranian primary school children. These data were transformed to determine the corneal position and orientation in 3-dimensional space, followed by a model fit that combines a biconic with a Zernike expansion. Girls were found to have slightly steeper corneas than boys. Both corneal surfaces show negative conic constants and significant higher-order aspheric Zernike terms. The corneal surfaces are decentered and misaligned with respect to each other and to the line of sight. Consequently, the average corneal surfaces may be considered as decentered and misaligned higher-order aspheres.
\end{abstract}

(c) 2018 Optical Society of America under the terms of the OSA Open Access Publishing Agreement

\section{Introduction}

As the cornea is the dominant refracting component of the eye, assessment of its age-related changes is crucial to understand either refractive development or the effect of aging on visual optics. It is generally accepted that the changes in optical quality of the eye are mostly due to changes of the crystalline lens, since it continues to develop throughout life [1,2]. The agerelated changes of the cornea, on the other hand, are much smaller and more difficult to determine as they are masked by the large inter-subject variability. In adults some studies reported that the mean corneal power either changes very little [3-5] or very gradually with age [6-9], and that the astigmatism axis changes from with-the-rule (WTR) in young eyes to against-the-rule (ATR) in older eyes [10-12]. Conic constants of the corneal surface (Q) also appear to change slightly with age [13], thus making the cornea more prolate, corresponding with a slight peripheral thinning with age $[4,7,9]$. These effects are small, however, and remain as yet unconfirmed [14].

The age-related changes in children, on the other hand, are more fundamental. This is especially apparent during early childhood, when the mean corneal curvature decreases rapidly by $4-6 \mathrm{D}$ until it stabilizes at adult values around the age of $2-3$ years $[3,15,16]$. This reduction is an important part of the emmetropization process, the gradual adjustment of the ocular components' growth rates to accomplish emmetropia (or mild hypermetropia) at 5 -6 years of age. Although no major corneal changes were reported after that age [17], it is conceivable that other, more subtle alterations may occur.

The goal of the current study is therefore to analyze the corneal shape changes in detail for schoolchildren aged between 6 and 12 years using a previously defined, realistic surface model [9]. This surface model enables a full, in-depth shape analysis based on elevation data. Moreover, it involves correcting for the misalignment between the topographer axis and the 
corneal axis and analyses how this changes with age. None of these things have ever been done before in children, as previous studies only reported standard measures such as corneal powers $(\mathrm{K} 1, \mathrm{~K} 2)$ and astigmatism. Both anterior and posterior surfaces will be assessed, along with the influence of gender, age and place of residence (urban or rural).

\section{Methods}

\subsection{Materials and Subjects}

The elevation and pachymetry maps used in this work were taken from the previously published Shahroud School Children Eye Cohort Study [18,19], The sampling method was explained in Refs. [18] and [19], and children were recruited in both rural and urban areas. The ethnicity of participants was Fars (97.2\%), Turkish (1.1\%) and others (1.7\%). During this study the Shahroud University of Medical Sciences (Shahroud, Iran) performed Pentacam (Oculus Optikgeräte, Wetzlar, Germany) Scheimpflug measurements on both eyes of 5601 schoolchildren among 5620 students who participated in the first phase of study. After applying the exclusion criteria (i.e. corneal diseases, history of surgery for ocular trauma, ptosis, congenital cataract and pterygium; and Pentacam results with errors) the remaining number of subjects was 4953 (2586 boys and 2367 girls) aged between 6 and 12.0 years (average $9.74 \pm 1.68$ years). The study adhered to the tenets of the Declaration of Helsinki and received approval of the ethical committee of the Shahroud University of Medical Sciences (Ref. no. 100/108054). Signed informed consent was obtained from the subjects' parents or guardians prior to testing and students participated willingly.

\subsection{Surface model}

Classic studies of the corneal shape [20,21] usually consider a simplistic surface model [22], consisting of prolate ellipsoid caps. A more realistic model, however, should also consider the fact that corneal surfaces are misaligned and decentered [23] high order aspheres [24]. Hence, an earlier theoretical model was applied [9], given by the following expression:

$$
z_{0}=\frac{c_{x} x_{0}^{2}+c_{y} y_{0}^{2}}{1+\sqrt{1-\left(1+Q_{x}\right)} c_{x}^{2} x_{0}^{2}+\left(1+Q_{y}\right) c_{y}^{2} y_{0}^{2}}+\sum_{j=0}^{J} a_{j} Z_{j}\left(x_{0}, y_{0}\right)+R_{V H O},
$$

where $z_{0}$ is the elevation. The first term corresponds to a biconic surface given by the two main curvatures $c_{x}=1 / R_{x}, c_{y}=1 / R_{y}$ (in $\mathrm{mm}$ ), and their corresponding dimensionless conic constants $Q_{x}, Q_{y}$. The second term is a Zernike polynomial expansion (ANSI Z80.28 standard, from 0 th up to 8 th order) and $R_{V H O}$ is a very high order residual.

To avoid potential redundancy between the three terms, a sequential data fit is applied as follows. First the biconic is fitted. Next, the residual (i.e. the difference between the best fit biconic and elevation data) is fitted to a 8th order Zernike polynomial. Finally, the resulting polynomial is subtracted to obtain the very high order residual $R_{V H O}$. This procedure ensures minimal redundancy since each term only applies to residuals that cannot be accounted for by previous terms.

Note that Equation 1 assumes a perfect alignment of the corneal surfaces to both the keratometric axis and the measuring instrument's axis, and that coordinate vector

$\mathbf{x}_{0}=\left(x_{0}, y_{0}, z_{0}\right)^{\mathrm{T}} \quad$ (with $\mathrm{T}$ denoting transposition) is defined with respect to the biconic's intrinsic coordinate system [24]. This assumption is not realistic, however, as corneal surfaces typically display an arbitrary position and orientation with respect to the keratometric axis. Moreover, the anterior and posterior surfaces can be decentered and misaligned with respect 
to each other. Consequently, the measured positions $\mathbf{x}=(x, y, z)^{\mathrm{T}}$ follow a rotated and displaced coordinate system with respect to the canonic coordinates $\mathrm{x} 0$ according to:

$$
\mathbf{x}=\mathbf{R}\left(\mathbf{x}_{\mathbf{0}}+\mathbf{d}\right),
$$

where $\mathbf{R}$ is a $3 \times 3$ unitary rotation matrix defined by tree Euler angles $(\alpha, \beta, \gamma)$ and a displacement vector $\mathbf{d}=\left(d_{x}, d_{y}, d_{z}\right)^{\mathrm{T}}$. Applying the affine transform leads to canonical equation (1). Due to Euler angle $\gamma$, the rotation around the $\mathrm{Z}$ axis (astigmatism axis), the main meridians are not aligned along the $\mathrm{X}$ and $\mathrm{Y}$ axes. The results for radii and conic constants are therefore given as $R_{\max }, Q_{\max }$ for the least step meridian (i.e. maximum radius $R$ ), and $R_{\min }$, $Q_{\min }$ for the steeper meridian. The affine transform ensures orthogonality between both meridians.

\subsection{Data fitting}

Pentacam elevation data consists of a matrix $\mathbf{Z}$ with samples $z_{i, j}$ defined on a regular square grid $\left(x_{i}, y_{j}\right)$ spaced $0.1 \mathrm{~mm}$ apart. Although the instrument's total field of view is $14 \times 14 \mathrm{~mm}$, so that the $\mathbf{Z}$ matrix has $141 \times 141$ elements, the number of valid data points is often substantially smaller. For every cornea the valid measured position vectors $\mathbf{x}_{i, j}=\left(x_{i}, y_{j}, z_{i, j}\right)$ of the anterior or posterior surface is fitted to the theoretical model of Equations 1 and 2. As the combination of these two equations leads to a highly complex expression, a simplified alternative implementation was developed consisting of several successive steps. This simplification uses a best-fit biconic and a best-fit general ellipsoid for a given surface instead, with approximately the same intrinsic axes. This way, the procedure of References [9] and [18] may be used to find the position and orientation of the 3 axes of the best-fit general ellipsoid. This starts from the canonical expression of the ellipsoid $\frac{x_{0}^{2}}{a^{2}}+\frac{y_{0}^{2}}{b^{2}}+\frac{z_{0}^{2}}{c^{2}}=0$, which can be rewritten in matrix form as

$$
\mathbf{x}_{0}^{\mathbf{T}} \Lambda \mathbf{x}_{0}=0,
$$

where is a $\Lambda$ diagonal matrix whose elements are the squared inverse of the semi-axes $\left(\frac{1}{a^{2}}, \frac{1}{b^{2}}, \frac{1}{c^{2}}\right)$. Applying Equation 2 then leads to the general expression:

$$
\mathbf{x}^{\mathrm{T}} \mathbf{R} \mathbf{\Lambda} \mathbf{R}^{\mathrm{T}} \mathbf{x}-\mathbf{2} \mathbf{x}^{\mathrm{T}} \mathbf{R} \mathbf{\Lambda} \mathbf{d}+a_{0}=0 .
$$

This general ellipsoid is a second-degree polynomial that may be solved by the simple and highly robust linear least-squares procedure [9]. The validity of this method was verified by demonstrating that the radii of curvature $R_{x}, R_{y}$ and conic constants $Q_{x}, Q_{y}$ of the best fit ellipsoid are comparable to those of the best fit biconic, meaning that the latter, more accurate model yields only a minor improvement over the ellipsoid fit (see Table 1). The main difference between both is that the biconic uses 4 independent parameters $\left(R_{x}, R_{y}, Q_{x}, Q_{y}\right)$, while the ellipsoid uses 3 parameters (semiaxes $a, b, c$ ). Consequently, the four parameters of the biconic cannot be completely independent from one another.

The fitting process starts from a custom best-fit sphere (i.e. an ellipsoid with $a=b=c=$ $R$ ), which has a nearly canonical expression. This still includes possible horizontal and vertical displacements of the apex, thus enabling a somewhat better fit. Next, the data fit consists of: 
1. Fitting a general ellipsoid to obtain the Euler angles $(\alpha, \beta, \gamma)$ and displacement vector $\mathbf{d}=\left(d_{x}, d_{y}, d_{z}\right)^{\mathrm{T}}$ of the surface's intrinsic coordinate system, as well as the initial estimates for the radii of curvature and conic constants $\left(R_{\max }, R_{\min }, Q_{\max }, Q_{\min }\right)$.

2. Obtaining the inverse of rotation matrix $\mathbf{R}$ by simple transposition to solve Equation 2 for $\mathbf{x}_{0}$ as $\mathbf{x}_{\mathbf{0}}=\mathbf{R}^{\mathrm{T}} \mathbf{x}-\mathbf{d}$. This affine transformation numerically aligns and centers the surface's intrinsic axes to the keratometric axis. After the affine transformation the principal meridians are aligned with the $\mathrm{X}_{0}$ and $\mathrm{Y}_{0}$ axes so $R_{x_{0}}=R_{\max }$, etc.

3. Fitting the biconic to the transformed data points to obtain the best fit biconic $(B F B)$.

4. Computing the residuals by subtracting the fitted biconic to the elevation data $(r=z-$ $B F B$ ) and fitting Zernike polynomials to the residual $r$. Since the resulting residual elevation values are low, these were multiplied by 1000 , converting Zernike coefficients $a_{j}$ and $R_{V H O}$ from $\mathrm{mm}$ to $\mu \mathrm{m}$.

5. Computing the remaining higher order residual $R_{V H O}=r-\sum_{j=0}^{J} a_{j} Z_{j}$ and obtain its root mean square (RMS) to estimate the goodness of the fit.

These fits were based on least-squares methods and used all available data points provided by the topographer (after coordinate normalization) to maximize the accuracy of the surface's position and orientation. The same set was also used to obtain the apical curvatures and conic constants. But since Zernike polynomials require data within a circular to form a complete and orthogonal basis, coefficients $a_{j}$ were determined inside a central area of $9 \mathrm{~mm}$ diameter, after coordinate normalization.

In summary, this model fit provides 6 parameters for position and orientation in the 3-D space, 4 biconic parameters and 45 Zernike coefficients. The goodness of fit was evaluated using the RMS residual, i.e. the distance between data and fitted model along the $z$ (keratometric) axis. The anterior and posterior corneal surfaces were analyzed independently and only right eyes were analyzed to avoid the influence of the mirror symmetry between left and right eyes of the same individual [25].

\subsection{Statistical analysis}

A key feature of this analysis is the removal of bad data points and outliers that can bias the analysis. To this end corneas with excessive RMS fitting errors were removed, defined as having an RMS error higher than the mean error plus three times its standard deviation. This left the anterior surface topographies of 2571 boys ( 15 removed) and 2357 girls ( 10 removed) and the posterior topographies of 2582 boys ( 4 removed) and 2363 girls (4 removed). The number of valid fits was therefore higher than $99 \%$ of all cases.

The descriptive statistical analysis of the main parameters consisted of computing the frequency histograms and the main statistical moments (mean, standard deviation, skewness and kurtosis), followed by a non-parametric Anderson-Darling test, supplemented by a QQplot analysis (not shown), the normality of their distributions. The Zernike coefficients were treated in much the same way, after determining those coefficients with a mean value significantly different from zero.

Finally, possible age-related changes were analyzed using linear regression, taking the effect of cluster sampling and stratification into account. This was done both using the entire cohort and after removing points outside the $95 \%$ confidence intervals of the linear regression (outliers).

\section{Results}

The area analyzed by the topographer was computed for each cornea, from which the equivalent diameter of a circle with the same area was estimated. Only the area with valid 
data points in both corneal surfaces was considered. For the 4953 corneas, the equivalent diameter ranged from $7.5 \mathrm{~mm}$ to $11.0 \mathrm{~mm}$; mean $9.9 \pm 0.47 \mathrm{~mm}$.

\subsection{RMS fit errors}

The fit errors of the general ellipsoid model are larger for the posterior surface than for the anterior surface by a factor 3.5 in boys and 3.7 in girls (Table 1, Figure 1). This indicates that the ellipsoid is better able to model the anterior surface as compared to the posterior. For the biconic model, on the other hand, the RMS fit error for the posterior surface improves by about one micrometer (11\%). Hence the biconic offers a better description of the posterior surface than the ellipsoid, while providing similar results for the anterior surface. The complete model of biconic plus Zernike polynomials provides the lowest RMS error of around one third of a micrometer for the anterior surface and roughly double that for the posterior surface. This is below the measurement error of the instrument [25].

Table 1. RMS fit error for the different corneal surface models

\begin{tabular}{llll}
\hline \multicolumn{4}{c}{ Boys } \\
\hline Surface & Ellipsoid & Biconic & Biconic + Zernikes \\
\hline Anterior & $2.63 \pm 0.77 \mu \mathrm{m}$ & $2.50 \pm 0.75 \mu \mathrm{m}$ & $0.37 \pm 0.06 \mu \mathrm{m}$ \\
Posterior & $9.21 \pm 2.36 \mu \mathrm{m}$ & $8.51 \pm 2.25 \mu \mathrm{m}$ & $0.69 \pm 0.22 \mu \mathrm{m}$ \\
\hline & \multicolumn{3}{c}{ Girls } \\
\hline Ellipsoid & Biconic & Biconic + Zernikes \\
\hline Anterior & $2.51 \pm 0.70 \mu \mathrm{m}$ & $2.36 \pm 0.54 \mu \mathrm{m}$ & $0.36 \pm 0.06 \mu \mathrm{m}$ \\
Posterior & $9.38 \pm 2.28 \mu \mathrm{m}$ & $8.62 \pm 2.14 \mu \mathrm{m}$ & $0.70 \pm 0.21 \mu \mathrm{m}$ \\
\hline
\end{tabular}

\subsection{Descriptive statistics}

Tables 2 summarizes the results for boys and girls, respectively. Only the radii of curvature passed the normality test, whereas the other parameters remained far from normality.

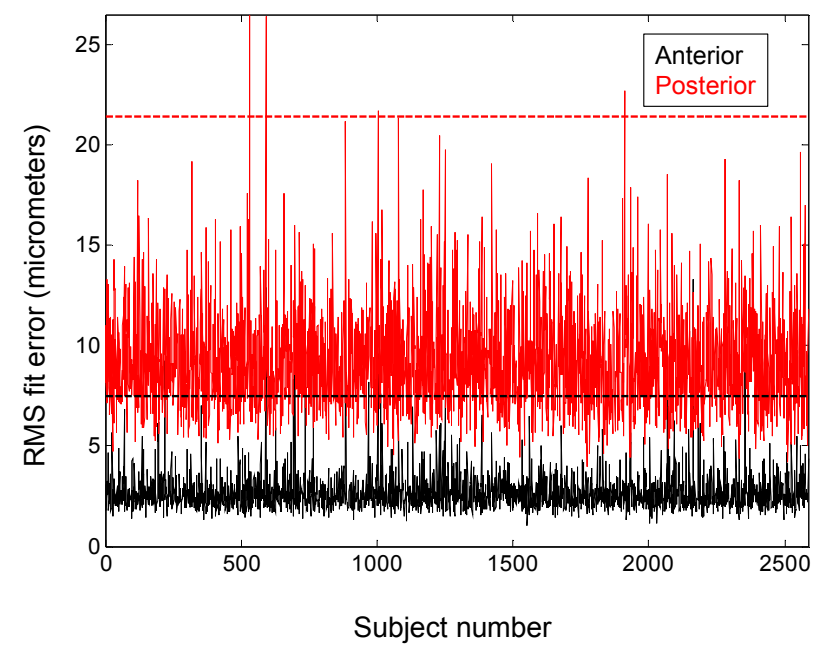

Fig. 1. RMS fit error for the general ellipsoid model (anterior and posterior) for boys. Fit errors were 3.5 times higher for the posterior surface (red). Dashed lines represent the cut-off thresholds used. 
The anterior surface of the cornea is a misaligned and centered higher-order asphere. Its conic constants were always negative (Table 2 ) with mean values around -0.41 , suggesting a prolate shape. This explains why the best-fit radii are higher than the apical (local) radii. Interestingly, these conic constants are not far from the optimal value $Q_{o p t}=-0.528$ that minimizes the spherical aberration of an elliptical cornea.20 The fourth and sixth order coefficients $\left(a_{4}^{0}\right.$ and $\left.a_{6}^{0}\right)$ are the only Zernike terms significantly different from zero. To a rough first approximation the Zernike optical aberration $\left(z_{n}^{m}\right)$ will be proportional to the aspherical terms of the surface $z_{n}^{m}=0.376 a_{n}^{m}$, with 0.376 being the difference in refractive index between cornea and air. Hence, one may expect that in typical corneas the 4th and 6th order spherical aberration to be around $z_{4}^{0} \approx 0.2 \mu \mathrm{m}$ and $z_{6}^{0} \approx-0.15 \mu \mathrm{m}$ respectively. The standard deviations are comparatively high, however, suggesting large inter-subject differences. For the radii of curvature, the standard deviations are much lower in relative terms (around 3\%). The difference between the maximum and minimum radii means that the apical astigmatism of the mean cornea is $0.68 \mathrm{D}$ for boys and $0.82 \mathrm{D}$ for girls.

The intrinsic axes of the anterior surface are displaced and rotated with respect to the instrument (keratometric) coordinate system. Figure 2 shows the frequency histogram of the third Euler angle $\gamma$, that is the rotation around the optical axis Z, for boys. This is the astigmatism axis. It can be seen that most corneas present with-the-rule astigmatism, between $-20^{\circ}$ and $+20^{\circ}$. Only $127(4.9 \%)$ boys and $70(3 \%)$ girls show against-the-rule astigmatism (anterior surface). For the posterior surface the against-the-rule is more frequent: 253 boys $(9.8 \%)$ and 221 girls $(9.5 \%)$.

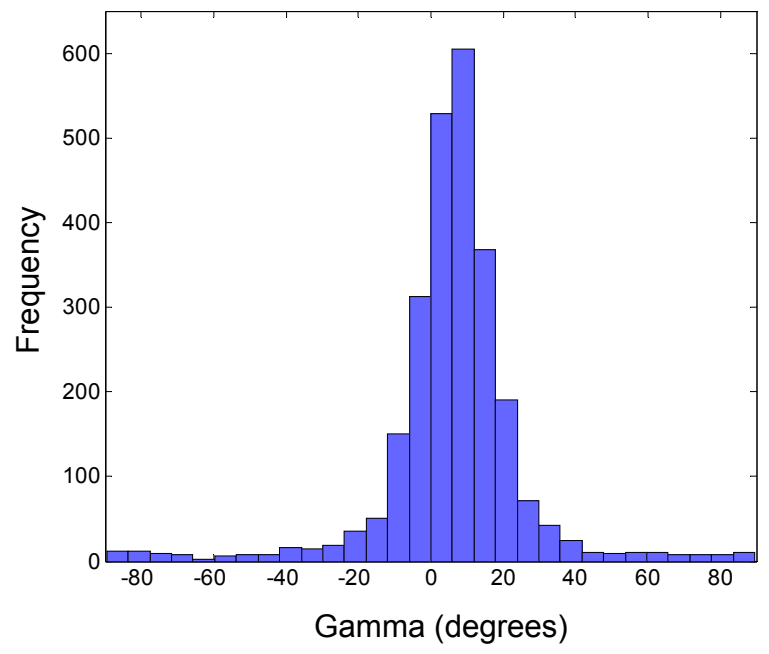

Fig. 2. Corneal gamma angle (astigmatism axis) histogram for the cohort of boys.

The tip and tilt angles of both anterior (blue) and posterior (red) are presented in Figure 3 for the cohort of boys, with the error bars crossing over the mean and showing the standard deviations. Despite the high inter-subject variability, the negative tip angle $-3.75^{\circ}$ (anterior) and $-6.40^{\circ}$ (posterior) is clearly significantly different from zero. The tilt angle is small for the anterior surface, but it is higher $\left(3.13^{\circ}\right)$ for the posterior surface. The apex coordinates $c_{x}$ and $c_{y}$ also suggest decentering (mainly horizontal). These values suggest that both surfaces are decentered and misalignment with respect to the keratometric axis, as well as to each other. 
Table 2: Descriptive statistics of the biconic parameters

\begin{tabular}{|c|c|c|c|c|c|}
\hline & \multicolumn{2}{|l|}{ Boys } & \multicolumn{2}{|l|}{ Girls } & \multirow[b]{2}{*}{$\begin{array}{c}\text { Boys vs } \\
\text { girls }^{\dagger}\end{array}$} \\
\hline & Mean \pm SD [Range] & Normal $^{*}$ & Mean \pm SD [Range] & Normal $^{*}$ & \\
\hline \multicolumn{6}{|c|}{ Anterior surface } \\
\hline BFS R (mm) & $8.07 \pm 0.24[7.23,8.57]$ & Yes & $7.92 \pm 0.23[7.04,8.35]$ & No & $<0.001$ \\
\hline $\mathrm{R}_{\max }(\mathrm{mm})$ & $7.79 \pm 0.26[6.90,8.72]$ & Yes & $7.66 \pm 0.26[6.78,8.55]$ & Yes & $<0.001$ \\
\hline $\mathrm{R}_{\min }(\mathrm{mm})$ & $7.67 \pm 0.27[6.73,8.56]$ & Yes & $7.52 \pm 0.26[6.65,8.56]$ & Yes & $<0.001$ \\
\hline $\mathrm{Q}_{\max }$ & $-0.41 \pm 0.10[-1.08,-0.06]$ & No & $-0.42 \pm 0.10[-0.84,-0.10]$ & No & 0.188 \\
\hline $\mathrm{Q}_{\min }$ & $-0.41 \pm 0.12[-1.11,-0.04]$ & No & $-0.43 \pm 0.11[-0.90,-0.06]$ & No & $<0.001$ \\
\hline $\mathrm{a}_{4}{ }^{0}(\mu \mathrm{m})$ & $0.54 \pm 0.47[-0.94,2.00]$ & No & $0.59 \pm 0.46[-0.85,2.01]$ & Yes & $<0.001$ \\
\hline $\mathrm{a}_{6}{ }^{0}(\mu \mathrm{m})$ & $-0.40 \pm 0.24[-1.18,0.38]$ & No & $-0.48 \pm 0.22[-1.18,0.22]$ & No & $<0.001$ \\
\hline $\operatorname{Apex~} c_{x}(m m)$ & $-0.51 \pm 0.29[-2.20,2.48]$ & No & $-0.51 \pm 0.26[-1.86,2.53]$ & No & 0.146 \\
\hline Apex $c_{y}(\mathrm{~mm})$ & $0.06 \pm 0.29[-1.60,1.21]$ & No & $0.08 \pm 0.27[-2.61,1.10]$ & No & 0.092 \\
\hline Tilt $\alpha^{\mathrm{o}}$ & $0.46 \pm 2.14[-9.95,10.2]$ & No & $0.57 \pm 2.11[-8.60,10.50]$ & No & 0.054 \\
\hline $\operatorname{Tip} \beta^{o}$ & $-3.75 \pm 1.89[-13.20,5.70]$ & No & $-3.76 \pm 1.93[-13.40,5.89]$ & No & 0.980 \\
\hline Axis $\gamma^{\mathrm{o}}$ & $6.10 \pm 19.60[-89.60,89.50]$ & No & $2.54 \pm 15.81[-89.70,83.10]$ & No & $<0.001$ \\
\hline \multicolumn{6}{|c|}{ Pachymetry } \\
\hline $\operatorname{Min}(\mu \mathrm{m})$ & $554.1 \pm 34.1[441,681]$ & No & $549.20 \pm 34.30[456,681]$ & No & $<0.001$ \\
\hline Vertex $(\mu \mathrm{m})$ & $558.6 \pm 34.0[454,687]$ & No & $553.70 \pm 34.10[459,683]$ & No & $<0.001$ \\
\hline \multicolumn{6}{|c|}{ Posterior surface } \\
\hline BFS R (mm) & $6.60 \pm 0.22[5.77,7.02]$ & Yes & $6.47 \pm 0.19[5.56,6.79]$ & No & $<0.001$ \\
\hline $\mathrm{R}_{\max }(\mathrm{mm})$ & $6.40 \pm 0.27[5.30,7.33]$ & Yes & $6.27 \pm 0.26[5.17,7.25]$ & Yes & $<0.001$ \\
\hline $\mathrm{R}_{\min }(\mathrm{mm})$ & $6.13 \pm 0.27[4.96,7.06]$ & Yes & $6.01 \pm 0.26[4.90,7.04]$ & Yes & $<0.001$ \\
\hline $\mathrm{Q}_{\max }$ & $-0.26 \pm 0.11[-0.94,0.05]$ & No & $-0.30 \pm 0.12[-0.84,0.02]$ & No & $<0.001$ \\
\hline $\mathrm{Q}_{\min }$ & $-0.39 \pm 0.14[-1.19,-0.04]$ & No & $-0.44 \pm 0.15[-1.05,-0.05]$ & No & $<0.001$ \\
\hline $\mathrm{a}_{4}{ }^{0}(\mu \mathrm{m})$ & $2.29 \pm 1.50[-2.50,6.51]$ & No & $2.63 \pm 1.39[-1.63,6.54]$ & No & $<0.001$ \\
\hline $\mathrm{a}_{6}{ }^{0}(\mu \mathrm{m})$ & $-0.81 \pm 0.78[-3.28,1.66]$ & No & $-0.99 \pm 0.84[-3.60,1.32]$ & No & $<0.001$ \\
\hline $\mathrm{a}_{6}^{2}(\mu \mathrm{m})$ & $0.81 \pm 0.64[-3.78,3.59]$ & No & $0.77 \pm 0.61[-2.92,3.08]$ & No & 0.002 \\
\hline $\mathrm{a}_{8}{ }^{0}(\mu \mathrm{m})$ & $-0.67 \pm 0.37[-2.76,0.57]$ & No & $-0.68 \pm 0.39[-2.26,0.35]$ & No & 0.656 \\
\hline $\operatorname{Apex} c_{x}(m m)$ & $-0.78 \pm 0.37[-3.64,2.26]$ & No & $-0.78 \pm 0.34[-4.02,2.64]$ & No & 0.466 \\
\hline Apex $c_{y}(\mathrm{~mm})$ & $0.37 \pm 0.42[-2.85,2.90]$ & No & $0.39 \pm 0.39[-3.79,2.06]$ & No & 0.305 \\
\hline Tilt $\alpha^{\mathbf{o}}$ & $3.13 \pm 3.93[-22.80,15.70]$ & No & $3.38 \pm 3.63[-19.10,14.50]$ & No & 0.027 \\
\hline $\operatorname{Tip} \beta^{\circ}$ & $-6.41 \pm 3.40[-23.40,10.60]$ & No & $-6.51 \pm 2.98[-21.50,11.50]$ & No & 0.542 \\
\hline Axis $\gamma^{\circ}$ & $4.92 \pm 25.57[-89.30,89.90]$ & No & $2.47 \pm 26.70[-89.30,90.00]$ & No & $<0.001$ \\
\hline
\end{tabular}

SD: Standard deviation. other symbols defined in the text.

*Anderson-Darling test for normality, supplemented by QQ plots (in bold)

${ }^{\dagger}$ Mann-Whitney U test, $\mathrm{p}<0.05$ indicates significant differences (in bold)

The pachymetry at the thinnest point is only slightly less than at the corneal vertex $(-4.5$ $\mu \mathrm{m})$, while boys' corneas are slightly thicker than those of girls $(+4.9 \mu \mathrm{m})$. Both differences only represent $1 \%$ of the mean value, however. 


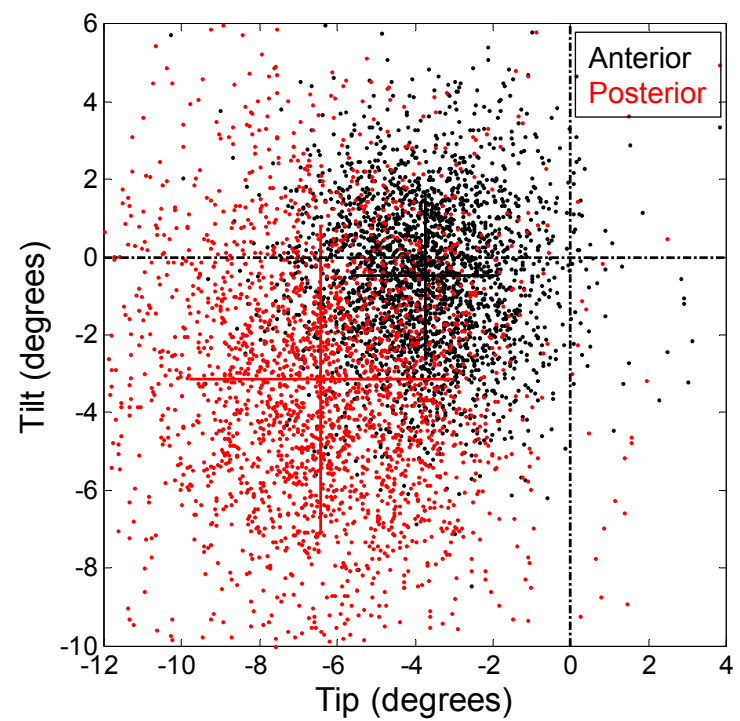

Fig.3. Corneal tip and tilt angles for the anterior (blue) and posterior surfaces (posterior) for boys.

Besides the well-known fact that the posterior surface has a steeper curvature than the anterior surface, these results suggest another main difference in that the posterior surface of the cornea cannot be as well described by the simple canonical ellipsoid model as the latter. This is reflected in many ways, such as e.g. the higher fit error of the ellipsoid model (see Figure 1 and Table 1). Moreover, the posterior surface Zernike coefficients are much higher in magnitude, and there are two additional coefficients $a_{6}^{2}=0.81 \pm 0.64 \mu \mathrm{m}, \quad a_{8}^{0}=-0.67 \pm 0.37$ $\mu \mathrm{m}$ (boys) that are significantly different from zero $(\mathrm{p}<0.05)$. This means that the back surface is an 8 th order asphere, and the term $a_{6}^{2}$ means more irregularity and departure from symmetry. Finally, the tip and tilt angles are much higher compared to the anterior surface, indicating more decentration and misalignment.
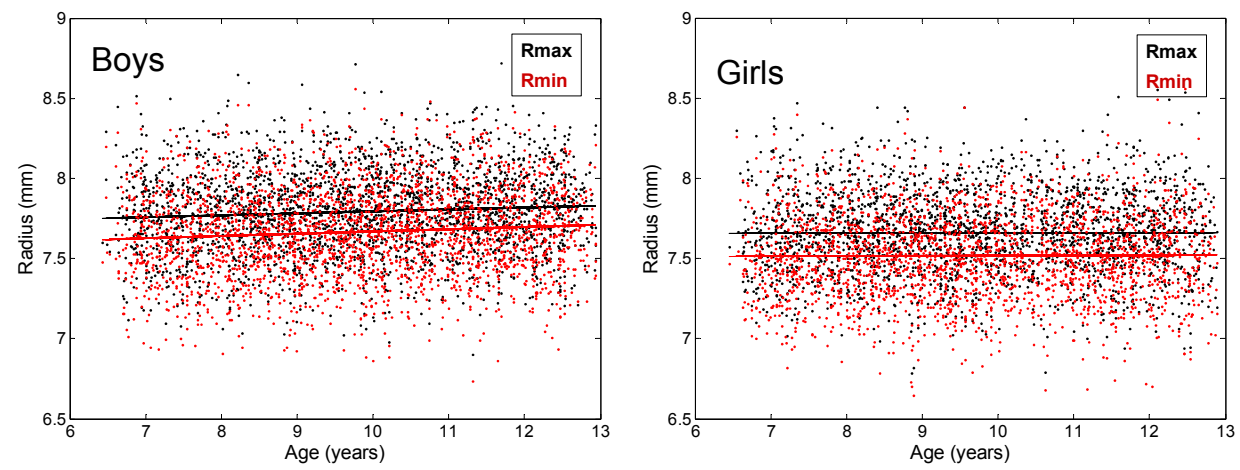

Fig.4. Changes of the curvature radii of the anterior surface as a function of age. 


\subsection{Multivariate analysis}

Assessing the influence of age, gender, body height and living location (urban or rural), it is seen that these factors contribute only modestly to the overall variance of the corneal shape parameters (Table 3). This is evidenced by the coefficients of determination $\mathrm{R}^{2}$ that remain very low in all cases, suggesting that the magnitude of the age-related changes is much lower than the inter-subject variability. Even though these effects are statistically significant (i.e. there is a high probability that these observations are real), they are usually small and probably of limited importance.

Only the anterior surface radii of curvature in boys show a modest increase with age (about $10 \mu \mathrm{m} /$ year, corresponding to a $0.5 \mathrm{D}$ power decrease from age 6-12). Meanwhile the corneal thickness $\left(2.34 \mu \mathrm{m} /\right.$ year) and $\mathrm{R}_{\max }$ for the posterior surface also increase, suggesting a continued, but almost negligible, growth in corneal size for boys, but not for girls (see Figure 4). Other statistically significant changes with age were also seen (Table 3), but their magnitudes are very small and unlikely to be relevant.

Boys were found to have slightly larger radii and thickness than girls (Table 2), suggesting a somewhat bigger cornea. Nevertheless, these differences are below $2 \%$ for radii and below $1 \%$ for corneal thickness. The surfaces in girls are also somewhat more aspherical, since both conic constants and higher order Zernike coefficients show are higher in magnitude compared to boys (Table 2).

The radii of curvature $R$ and conic constants also depended significantly on living location, but here too the effects were very modest with urban children having marginally steeper posterior corneas and slightly larger conic constants (Table 3). Body height had a larger influence, with taller children generally having flatter corneas.

\section{Discussion}

The mean apical radii of the anterior surface $\left(R_{\max }=7.79 \pm 0.26 \mathrm{~mm}\right.$ and $R_{\min }=7.67 \pm 0.27$ $\mathrm{mm}$ in boys) were slightly lower than standard values from the literature $[17,26]$, possibly due to the fact that they correspond to the point of maximum curvature (apex) whereas standard values are given for the vertex normal [23]. The global average of the apical radii, combining both boys and girls, were $R_{\max }=7.73 \mathrm{~mm}$ and $R_{\min }=7.60 \mathrm{~mm}$, which is slightly lower than the average values found previously for Caucasian adults $(7.69 \pm 0.26 \mathrm{~mm}$ and $7.54 \pm 0.27 \mathrm{~mm}$ ) [9]. The latter study also reported a gradual decrease of the radii with age, as well as nearly identical standard deviations as the ones found here. The posterior surface averages were $R_{\max }=6.34 \pm 0.27 \mathrm{~mm}$ and $R_{\min }=6.07 \pm 0.27 \mathrm{~mm}$, which are also close to the values of Caucasian adults $(6.20 \pm 0.26 \mathrm{~mm}$ and $5.99 \pm 0.28 \mathrm{~mm})$. Similarly, a close agreement was seen between the average anterior conic constants of this study ( -0.41 for boys and -0.42 for girls) and the values in European adults [9] (-0.40 and -0.41 , respectively), but not for the conic constants of the posterior surface ( -0.35 and -0.56 , respectively). But since from the eight parameters of the anterior and posterior biconic surfaces the posterior surface conic constants were the least relevant for optical performance, the optical performance in both groups is expected to be basically the same. The change may therefore point at a peripheral corneal thinning in these children, which was reported before in Caucasian adults [7].

The conic constants reported here are more negative compared to those in most of earlier studies and no oblate corneas were found within this population. As discussed before [9] it is well-known that the cornea seems to become more prolate (more negative $\mathrm{Q}$ values) for larger measurement areas [32,33]. More negative conic constants may therefore be the results of considering larger corneal diameters than the other works. 
Table 3. Standardized beta coefficients for multivariate regression Beta (p)*

\begin{tabular}{|c|c|c|c|c|c|c|}
\hline & & Age & Gender & Height & Location & $\mathrm{R}^{2}(\mathrm{p})$ \\
\hline \multirow[t]{2}{*}{ BFS R } & Ant. & $\begin{array}{c}-0.192 \\
(<0.001)^{\dagger}\end{array}$ & $\begin{array}{c}-0.236 \\
(<0.001)^{\dagger}\end{array}$ & $\begin{array}{c}0.283 \\
(<0.001)^{\dagger}\end{array}$ & $\begin{array}{c}0.010 \\
(0.451)\end{array}$ & $\begin{array}{c}0.082 \\
(<0.001)\end{array}$ \\
\hline & Post. & $\begin{array}{c}-0.194 \\
(<0.001)^{\dagger}\end{array}$ & $\begin{array}{c}-0.161 \\
(<0.001)^{\dagger}\end{array}$ & $\begin{array}{c}0.278 \\
(<0.001)^{\dagger}\end{array}$ & $\begin{array}{c}0.031 \\
(0.029)^{\dagger}\end{array}$ & $\begin{array}{c}0.052 \\
(<0.001)\end{array}$ \\
\hline \multirow[t]{2}{*}{$\mathrm{R}_{\max }$} & Ant. & $\begin{array}{c}-0.152 \\
(<0.001)^{\dagger}\end{array}$ & $\begin{array}{c}-0.244 \\
(<0.001)^{\dagger}\end{array}$ & $\begin{array}{c}0.252 \\
(<0.001)^{\dagger}\end{array}$ & $\begin{array}{c}0.011 \\
(0.449)\end{array}$ & $\begin{array}{c}0.083 \\
(<0.001)\end{array}$ \\
\hline & Post. & $\begin{array}{c}-0.152 \\
(<0.001)^{\dagger}\end{array}$ & $\begin{array}{c}-0.239 \\
(<0.001)^{\dagger}\end{array}$ & $\begin{array}{c}0.246 \\
(<0.001)^{\dagger}\end{array}$ & $\begin{array}{c}0.069 \\
(<0.001)^{\dagger}\end{array}$ & $\begin{array}{c}0.081 \\
(<0.001)\end{array}$ \\
\hline \multirow[t]{2}{*}{$\mathrm{R}_{\min }$} & Ant. & $\begin{array}{c}-0.093 \\
(<0.001)^{\dagger}\end{array}$ & $\begin{array}{c}-0.266 \\
(<0.001)^{\dagger}\end{array}$ & $\begin{array}{c}0.188 \\
(<0.001)^{\dagger}\end{array}$ & $\begin{array}{c}0.033 \\
(0.018)^{\dagger}\end{array}$ & $\begin{array}{c}0.086 \\
(<0.001)\end{array}$ \\
\hline & Post. & $\begin{array}{c}-0.154 \\
(<0.001)^{\dagger}\end{array}$ & $\begin{array}{c}-0.224 \\
(<0.001)^{\dagger}\end{array}$ & $\begin{array}{c}0.171 \\
(<0.001)^{\dagger}\end{array}$ & $\begin{array}{c}0.036 \\
(0.010)^{\dagger}\end{array}$ & $\begin{array}{c}0.060 \\
(<0.001)\end{array}$ \\
\hline \multirow[t]{2}{*}{$\mathrm{Q}_{\max }$} & Ant. & $\begin{array}{c}0.214 \\
(<0.001)^{\dagger}\end{array}$ & $\begin{array}{l}-0.023 \\
(0.104)\end{array}$ & $\begin{array}{c}-0.191 \\
(<0.001)^{\dagger}\end{array}$ & $\begin{array}{c}0.028 \\
(0.049)^{\dagger}\end{array}$ & $\begin{array}{c}0.018 \\
(<0.001)\end{array}$ \\
\hline & Post. & $\begin{array}{c}0.067 \\
(0.007)^{\dagger}\end{array}$ & $\begin{array}{c}-0.186 \\
(<0.001)^{\dagger}\end{array}$ & $\begin{array}{c}-0.072 \\
(0.004)^{\dagger}\end{array}$ & $\begin{array}{c}0.075 \\
(<0.001)^{\dagger}\end{array}$ & $\begin{array}{c}0.042 \\
(<0.001)\end{array}$ \\
\hline \multirow[t]{2}{*}{$\mathrm{Q}_{\min }$} & Ant. & $\begin{array}{c}0.218 \\
(<0.001)^{\dagger}\end{array}$ & $\begin{array}{c}-0.072 \\
(<0.001)^{\dagger}\end{array}$ & $\begin{array}{c}-0.170 \\
(<0.001)^{\dagger}\end{array}$ & $\begin{array}{c}0.037 \\
(0.010)^{\dagger}\end{array}$ & $\begin{array}{c}0.023 \\
(<0.001)\end{array}$ \\
\hline & Post. & $\begin{array}{c}0.052 \\
(0.038)^{\dagger}\end{array}$ & $\begin{array}{c}-0.153 \\
(<0.001)^{\dagger}\end{array}$ & $\begin{array}{c}-0.117 \\
(<0.001)^{\dagger}\end{array}$ & $\begin{array}{c}0.026 \\
(0.070)\end{array}$ & $\begin{array}{c}0.031 \\
(<0.001)\end{array}$ \\
\hline \multirow[t]{2}{*}{ Tilt $\alpha^{\mathrm{o}}$} & Ant. & $\begin{array}{c}0.026 \\
(0.308)\end{array}$ & $\begin{array}{l}-0.028 \\
(0.050)\end{array}$ & $\begin{array}{c}-0.060 \\
(0.018)^{\dagger}\end{array}$ & $\begin{array}{c}0.023 \\
(0.116)\end{array}$ & $\begin{array}{c}0.004 \\
(0.004)\end{array}$ \\
\hline & Post. & $\begin{array}{c}0.006 \\
(0.827)\end{array}$ & $\begin{array}{c}-0.036 \\
(0.011)^{\dagger}\end{array}$ & $\begin{array}{l}-0.020 \\
(0.440)\end{array}$ & $\begin{array}{c}0.020 \\
(0.162)\end{array}$ & $\begin{array}{c}0.002 \\
(0.071)\end{array}$ \\
\hline $\operatorname{Tip} \beta^{\circ}$ & Ant. & $\begin{array}{c}0.116 \\
<0.001)^{\dagger}\end{array}$ & $\begin{array}{c}0.001 \\
(0.935)\end{array}$ & $\begin{array}{l}-0.036 \\
(0.160)\end{array}$ & $\begin{array}{c}-0.038 \\
(0.008)^{\dagger}\end{array}$ & $\begin{array}{c}0.010 \\
(<0.001)\end{array}$ \\
\hline \multirow[t]{2}{*}{ Apex $c_{x}$} & Ant. & $\begin{array}{c}0.126 \\
(<0.001)^{\dagger}\end{array}$ & $\begin{array}{c}0.019 \\
(0.174)\end{array}$ & $\begin{array}{c}-0.055 \\
(0.030)^{\dagger}\end{array}$ & $\begin{array}{c}-0.039 \\
(0.007)^{\dagger}\end{array}$ & $\begin{array}{c}0.010 \\
(<0.001)\end{array}$ \\
\hline & Post. & $\begin{array}{c}0.059 \\
(0.019)^{\dagger}\end{array}$ & $\begin{array}{c}0.009 \\
(0.526)\end{array}$ & $\begin{array}{l}-0.017 \\
(0.493)\end{array}$ & $\begin{array}{l}-0.016 \\
(0.279)\end{array}$ & $\begin{array}{c}0.003 \\
(0.029)\end{array}$ \\
\hline Apex $c_{y}$ & Ant. & $\begin{array}{l}-0.028 \\
(0.270)\end{array}$ & $\begin{array}{c}0.025 \\
(0.078)\end{array}$ & $\begin{array}{c}0.065 \\
(0.010)^{\dagger}\end{array}$ & $\begin{array}{l}-0.024 \\
(0.097)\end{array}$ & $\begin{array}{c}0.004 \\
(0.002)\end{array}$ \\
\hline$a_{8}^{0}$ & Post. & $\begin{array}{l}-0.004 \\
(0.865)\end{array}$ & $\begin{array}{l}-0.019 \\
(0.184)\end{array}$ & $\begin{array}{c}0.081 \\
(0.001)^{\dagger}\end{array}$ & $\begin{array}{c}0.018 \\
(0.211)\end{array}$ & $\begin{array}{c}0.006 \\
(<0.001)\end{array}$ \\
\hline Pachy $_{\text {min }}$ & & $\begin{array}{c}-0.066 \\
(0.009)^{\dagger}\end{array}$ & $\begin{array}{c}-0.073 \\
\left(<0.001^{\dagger}\right.\end{array}$ & $\begin{array}{l}-0.020 \\
(0.418)\end{array}$ & $\begin{array}{c}-0.133 \\
(<0.001)^{\dagger}\end{array}$ & $\begin{array}{c}0.028 \\
(<0.001)\end{array}$ \\
\hline Pachy $_{\text {vertex }}$ & & $\begin{array}{c}-0.066 \\
(0.008)^{\dagger}\end{array}$ & $\begin{array}{c}-0.072 \\
(<0.001)^{\dagger}\end{array}$ & $\begin{array}{l}-0.023 \\
(0.365)\end{array}$ & $\begin{array}{c}-0.132 \\
(<0.001)^{\dagger}\end{array}$ & $\begin{array}{c}0.028 \\
(<0.001)\end{array}$ \\
\hline
\end{tabular}

* Showing only regressions with significant coefficients and normally distributed residuals

${ }^{\dagger}$ Significant at $\mathrm{p}<0.05$

Current results are also near those from earlier reports in the literature that used a more basic aspherical model (Table 4), provided the diameter of the analysis is considered. The Orinda Longitudinal study of Myopia [27] reported some longitudinal changes in $Q$ and $R$ that made the cornea more oblate with age. This could not be confirmed in current crosssectional study. 
Table 4: Comparison with the literature

\begin{tabular}{|c|c|c|c|c|c|c|c|}
\hline Author(year) & Country & $\mathbf{N}$ & Age & Diameter & Meridian & $\mathbf{R}(\mathbf{m m})$ & $\mathbf{Q}$ \\
\hline \multirow[t]{2}{*}{ Lam (1999) [28] } & Hong Kong & 142 & $6-17$ & 9 & Hor. & $7.84 \pm 0.26$ & $-0.18 \pm 0.15$ \\
\hline & & & & & Vert. & $7.67 \pm 0.27$ & NA \\
\hline Davis (2005) [27] & USA & 72 & $6-15$ & 8 & NA & $7.55 \pm 0.24$ & $-0.35 \pm 0.10$ \\
\hline \multirow[t]{2}{*}{ Chan (2008) [29] } & Hong Kong & 73 & $6-15$ & 9.8 & Flat & $7.80 \pm 0.22$ & NA \\
\hline & & & & & Steep & $7.53 \pm 0.19$ & $-0.42 \pm 0.13$ \\
\hline $\begin{array}{c}\text { Zhang (2011) } \\
{[30]}\end{array}$ & China & 31 & $6-10$ & 6 & NA & NA & $-0.32 \pm 0.12$ \\
\hline \multirow[t]{2}{*}{ Chan (2011) [31] } & Hong Kong & 217 & $6-12$ & 9 & Flat & $7.85 \pm 0.24$ & $-0.44 \pm 0.11$ \\
\hline & & & & & Steep & $7.59 \pm 0.26$ & $-0.22 \pm(\mathrm{NA})$ \\
\hline \multirow[t]{2}{*}{ Current } & Iran & 4953 & $6-12$ & $7-11$ & Flat & $7.73 \pm 0.26$ & $-0.42 \pm 0.10$ \\
\hline & & & & & Steep & $7.60 \pm 0.27$ & $-0.42 \pm 0.12$ \\
\hline
\end{tabular}

NA: Not available

The fact that the cornea becomes more prolate for increasing corneal diameters suggests that the biconic model is too simplistic, which is also supported by the fact that the model consistently leads to Zernike coefficients $a_{4}^{0}$ and $a_{6}^{0}$ that are significantly different from zero. This means that the corneal surfaces are higher-order aspheres. For the anterior surface the values found here $\left(a_{4}^{0}=0.54 \pm 0.47 \mu \mathrm{m}, a_{6}^{0}=-0.40 \pm 0.24 \mu \mathrm{m}\right)$ are again close to the values found in young Caucasian adults [9]. For the posterior surface relevant differences were also found, both in the value of the Zernike coefficients, as well in the additional orders $a_{6}^{2}$ and $a_{8}^{0}$ that are significantly different from zero. These differences may be the result of differences in ethnicity, age, the range (age ranges of 75 versus 6 years), or sample size (400 versus 5000 subjects) between both studies. The significant results found in the current study may therefore also be present in the Caucasian adult study, but masked by a larger inter-subject variability within a substantially smaller population.

The misalignment and decentration of the surfaces is also consistent with previous studies (Table 2) $[23,9]$ but again compared to Caucasian adults mean values deviate by \pm 1 standard deviation. The anterior surface has a statistically significant tip angle with an associated horizontal decentering and misalignment with the keratometric axis, while the posterior surface has a higher tip angle and a higher tilt. The posterior surface of the cornea is therefore misaligned with respect to the anterior surface.

On average, the surfaces of these children appear to be a pair of regular, non-rotationally symmetric, misaligned and decentered, general aspheres. Here these aspheres are the sum of a biconic, a limited number of significant Zernike polynomials, and an irregular residual given by the rest of (non-significant) Zernike coefficients. The posterior surface deviates more from the centered and aligned sphere than the anterior surface as it shows not only higher magnitudes in higher order terms, but also additional significant coefficients, leading it to be more misaligned and decentered than the anterior surface and having a higher irregular residual. Perhaps this might be explained by more endothelial surface irregularities or measurement errors, which are expected to be higher for the posterior surface as the Scheimpflug technique obtains an image through the anterior cornea.

During the primary school period (between ages 6 and 12) the girls group do not show any significant corneal size changes, suggesting that their corneas are already mature. In the same period boys show a small residual size increase, corresponding to less than $1 \%$ in thickness 
and radii of curvature. Taller children tended to have flatter corneas, while living location only had a very modest influence on the corneal shape in this cohort.

\section{Funding and disclosures}

\subsection{Funding}

Shahroud School Children Eye Cohort Study is funded by the Noor Ophthalmology Research Center and Shahroud University of Medical Sciences (project No. 9329).

\subsection{Disclosures}

The authors declare that there are no conflicts of interest related to this article.

\section{References}

1. A. Glasser and M. C. Campbell, "Presbyopia and the optical changes in the human crystalline lens with age," Vis.Res. 38(2), 209-229. (1998).

2. M. Dubbelman, G. L. van der Heijde and H. A. Weeber, "Change in shape of the aging human crystalline lens with accommodation," Vis. Res. 45(1), 117-132 (2005).

3. R. A. Gordon and P. B. Donzis, "Refractive development of the human eye," Arch. Ophthalmol. 103(6),785789 (1985).

4. H. Hashemi, S. Asgari,M. H. Emamian, S. Mehravaran and A. Fatouhi, "Age-Related Changes in Corneal Curvature and Shape: The Shahroud Eye Cohort Study," Cornea. 34(11),1456-1458 (2015).

5. C. S. Ooi and T. Grosvenor, "Mechanisms of emmetropization in the aging eye," Optom. Vis. Sci. 72(2), 60-66 (1995).

6. K. Hayashi, H. Hayashi and F. Hayashi, "Topographic analysis of the changes in corneal shape due to aging," Cornea 14(5), 527-532 (1995).

7. M. Dubbelman, V. A. Sicam and G. L. van der Heijde, "The shape of the anterior and posterior surface of the aging human cornea," Vis. Res. 46(6-7), 993-1001 (2006).

8. H. Topuz, M. Ozdemir A. Cinal and Y. Gumusalam, "Age-related differences in normal corneal topography," Ophthalmic Surg. Lasers Imaging Retina 2004; 35(4), 298-303 (2004).

9. R. Navarro, J. J. Rozema and M. J. Tassignon, "Optical changes of the human cornea as a function of age," Optom. Vis. Sci. 90(6): 587-598 (2013).

10. E. Gudmundsdottir, F. Jonasson, V. Jonsson, E. Stefanson, H. Sasaki and K. Sasaki, "'With the rule" astigmatism is not the rule in the elderly. Reykjavik Eye Study: a population based study of refraction and visual acuity in citizens of Reykjavik 50 years and older," Acta Ophthalmol. Scand. 78(6), 642-646 (2000).

11. T. W. Leung, A. K. Lam, L. Deng L and C. Kee, "Characteristics of astigmatism as a function of age in a Hong Kong clinical population,” Optom. Vis. Sci. 89(7), 984-992 (2012).

12. T. Goto, S. D. Klyce, X. Zheng, N. Maeda, T. Kuroda and C. Ide, "Gender- and age-related differences in corneal topography," Cornea 20(3) 270-276 (2001).

13. G. H. Beiko, W. Haigis and A.Steinmueller, "Distribution of corneal spherical aberration in a comprehensive ophthalmology practice and whether keratometry can predict aberration values," J. Cataract. Refract. Surg. 33(5) 848-858 (2007).

14. K. Scholz, A. Messner, T. Eppig, H. Bruenner and A. Langenbucher, "Topography-based assessment of anterior corneal curvature and asphericity as a function of age, sex, and refractive status," J. Cataract Refract. Surg. 35(6) 1046-1054 (2009).

15. P. A. Asbell, B. Chiang, M. E. Somers and K. S. Morgan, "Keratometry in children," CLAO J. 16(2) 99-102 (1990).

16. Y. Inagaki, "The rapid change of corneal curvature in the neonatal period and infancy," Arch. Ophthalmol.; 104(7) 1026-1027 (1986).

17. K. Zadnik, R. E. Manny, J. A. Yu, G. L Mitchell, et al. , "Ocular component data in schoolchildren as a function of age and gender," Optom.Vis. Sci. 80(3):226-236 (2003).

18. H. Hashemi, M. Khabazkhoob, A. Asharlous, A. Yekta, M. H. Emamian and A. Fotouhi, "Overestimation of hyperopia with autorefraction compared with retinoscopy under cycloplegia in school-age children," $\mathrm{Br}$. J. Ophthalmol. 2018; doi: 10.1136/bjophthalmol-2017-311594.

19. H. Hashemi, R. Pakzad, R. Iribarren, M. Khabazkhoob, M. H. Emamian and A. Fotouhi, "Lens power in Iranian schoolchildren: a population-based study," Br. J. Ophthalmol. 102(6) 779-783 (2017).

20. D. A. Atchison and G. Smith, "Optics of the Human Eye," Butterworth Heineman (Boston, 2000).

21. P. M. Kiely, G. Smith and L. G. Carney, "The mean shape of the human cornea," Optica Acta 29(8) 1027-1040 (1982).

22. M. Guillon, D. P. Lydon and C. Wilson, "Corneal topography: a clinical model," Ophthalmic Physiol. Opt. 6(1) 47-56 (1986). 
23. R. B. Mandell, C. S. Chiang and S. A. Klein, "Location of the major corneal reference points," Optom. Vis. Sci. 72(11) 776-784 (1995).

24. R. Navarro, L. González and J. L. Hernandez, "Optics of the average normal cornea from general and canonical representations of its surface topography,” J. Opt. Soc. Am. A 23(2) 219-232 (2006).

25. P. Rodríguez, R. Navarro and J. J. Rozema, "Eigencorneas: application of principal component analysis to corneal topography," Ophthalmic Physiol. Opt. 34(6) 667-677 (2014).

26. H. C. Fledelius and M. Stubgaard, "Changes in refraction and corneal curvature during growth and adult life," Acta Ophthalmol. 64(5) 487-491 (1986).

27. W. R. Davis, T. W. Raasch, G. L.Mitchell, D. O. Mutti and K. Zadnik , "Corneal asphericity and apical curvature in children: a cross-sectional and longitudinal evaluation,” Invest. Ophthalmol. Vis. Sci. 46(6) 1899$1906(2005)$

28. C. Lam, M. Edwards and M. Millodot, "A 2-Year Longitudinal Study of Myopia Progression and Optical Component Changes,” Optom. Vis. Sci. 76(6) 370-80 (1999).

29. B. Chan, P. Cho and S. W. Cheung, "Orthokeratology practice in children in a university clinic in Hong Kong," Clin. Exp. Optom. 91(5) 453-460 (2008).

30. Z. Zhang, J. Wang, W. Niu , "Corneal asphericity and its related factors in 1052 Chinese subjects," Optom. Vis. Sci. 88(10) 1232-1239 (2011)

31. K. Y. Chan, S. W. Cheung and P. Cho, "Corneal parameters of six-to 12-year-old Chinese children," Clin. Exp. Optom. 95(2) 160-165 (2012).

32. J. M. González-Méijome, C. Villa-Collar, R. Montés-Micó and A. Gomes, "Asphericity of the anterior human cornea with different corneal diameters," J. Cataract Refract. Surg. 33(3) 465-473 (2007).

33. M. A. Rosales, M. Juárez-Aubry, E. López-Olazagasti, J. Ibarra and E. Tepichín, "Anterior corneal profile with variable asphericity," Appl. Opt.; 48(35) 6594-6599 (2009). 